\title{
ATENÇÃO À DEPENDÊNCIA QUÍMICA NA UNIVERSIDADE FEDERAL DO ESPÍRITO SANTO: POSSIBILIDADES DA EXTENSÃO UNIVERSITÁRIA
}

\author{
Substance Dependence Care at Federal University \\ of Espírito Santo: Program Extension Possibilities \\ Atención a la Dependencia Química en la Universidad \\ Federal del Espírito Santo: Posibilidad de la Extensión Universitaria
}

Thiago Rodrigues de Amorim

Welington Serra Lazarini ${ }^{2}$

Marluce Miguel de Siqueira ${ }^{3}$

\begin{abstract}
Resumo
Objetiva-se neste trabalho descrever dois programas permanentes de extensão da Universidade Federal do Espírito Santo que atuam com base nos princípios de ensino, pesquisa, assistência e extensão, direcionados à temática das drogas: o Núcleo de Estudos sobre o Álcool e outras Drogas e o Programa de Atendimento ao Alcoolista do Hospital Universitário Cassiano Antônio Moraes. Tais estratégias constituem referências no Estado do Espírito Santo, promovendo a produção e divulgação de conhecimentos, a realização de pesquisas, a cooperação técnica e assessoria no campo da dependência química, a colaboração na organização de práticas de saúde que atendam às necessidades da população e a assistência ambulatorial para o tratamento do alcoolismo. Inicialmente descrevem-se o Programa de Atendimento ao Alcoolista e sua atuação interdisciplinar no atendimento a pacientes alcoolistas. Em seguida, são apresentadas a origem, a organização e as competências do Núcleo de Estudos sobre o Álcool e outras Drogas.
\end{abstract}

Palavras-chave: Alcoolismo. Tabagismo. Drogas ilícitas. Educação em saúde. Relações Comunidade-Instituição.

\begin{abstract}
The objective of this paper is to describe two permanent extension programs carried out by the Federal University of Espírito Santo that are based on research, assistance and learning focused on the thematic of drug abuse. They are the Drugs and Alcohol Study Center and the Assistance Program for Alcoholics based at the University Hospital Cassiano Antônio Moraes. These strategies are considered as references in Espírito Santo by promoting research, diffusion of knowledge, technique cooperation, consulting on substance abuse, assisting on the organization of health practices that address population's needs as well as providing ambulatory care for the treatment of alcoholism. On the first part of the paper, the Assistance Program for Alcoholics and its interdisciplinary approach to patients are described; then comes the description of the origins, organization and the attributions of Drugs and Alcohol Study Center.
\end{abstract}

Keywords: Alcoholism. Smoking. Street Drugs. Health Education. Community-Institutional Relations.

\section{Resumen}

El objetivo de este trabajo es describir dos programas permanentes de extensión de la Universidad Federal del Espírito Santo que actúan con base en los principios de la enseñanza, investigación, asistencia y extensión, en dirección al tema de las drogas: el Núcleo de Estudios sobre el Alcohol y otras Drogas y el Programa de Atención al Alcohólico del Hospital Universitario Cassiano Antônio Moraes. Dichas estrategias constituyen referencias en el Estado del Espírito Santo, promoviendo la producción y divulgación de conocimientos, la realización de investigaciones, la cooperación técnica y asesoría en el campo de la dependencia química, la colaboración en la organización de prácticas de salud que atiendan a las necesidades de la población y la asistencia en ambulatorio para el tratamiento del alcoholismo. Primeramente se describe el Programa de Atención al Alcohólico y su actuación interdisciplinaria en la atención a los pacientes alcohólicos. Enseguida, son presentados el origen, organización y las competencias del Núcleo de Estudios sobre el Alcohol y otras Drogas.

Palabras clave: Alcoholismo. Tabaquismo. Drogas ilícitas. Educación en Salud. Relaciones Comunidad-Institución. 


\section{INTRODUÇÃO}

0 consumo de substâncias que podem produzir alterações mentais confunde-se com a história da humanidade. Ao longo do tempo, diferentes grupos de pessoas passaram a associar essas substâncias a contextos variados, entre outros, festas e comemorações, rituais religiosos e tratamento de doenças.

Embora o termo droga se preste a várias interpretações, comumente suscita a idéia de uma substância proibida de uso ilegal e nocivo; contudo, considera-se como toda e qualquer substância, natural ou sintética, que, introduzida no organismo, modifica suas funções. Além disso, várias dessas substâncias têm o potencial de induzir, em algumas pessoas, um padrão de consumo problemático e com perda de controle denominado dependência. Essa dependência não acontece com todos os usuários, mas, quando ocorre, pode ser entendida como uma doença. Paradoxalmente, as drogas podem tanto ser úteis no tratamento de doenças quanto geradoras de doença. Assim, os conceitos que uma pessoa tem com relação a estas substâncias podem ser muito diferentes, dificultando, muitas vezes, a ajuda e/ou intervenção, em casos de transtornos relacionados às mesmas.

As drogas são classificadas em depressoras, estimulantes e perturbadoras das atividades mentais. De outro modo, podem ser categorizadas em legais - socialmente aceitas e difundidas no mercado e ilegais - prejudiciais à saúde humana, quando utilizadas em uma dosagem acima da capacidade metabólica do indivíduo.

Dentre aquelas lícitas consumidas no Brasil, destaca-se 0 álcool, responsável por vários transtornos, desde o abuso e dependência até a violência gerada, sobretudo, nas famílias e no trânsito. Conseqüentemente, estratégias de atenção à dependência química são necessárias, à luz da Política Nacional Antidrogas (PNAD), para que o enfrentamento da questão das drogas possa ser ampliado através de medidas eficazes que envolvam e contemplem todos os setores da sociedade. Neste sentido, a Universidade Federal do Espírito Santo, desenvolve importantes estratégias de produção de conhecimento e atenção à dependência química desde o ano de 1985.

Portanto, traçamos como objetivo descrever o Programa de Atendimento ao Alcoolista (PAA) e o Núcleo de Estudos sobre o Álcool e Outras Drogas (NEAD) - estratégias desenvolvidas na Universidade voltadas para atenção à dependência química, ressaltando também as contribuições tanto para a formação acadêmica quanto para a população atendida.

\section{PROGRAMA DE ATENDIMENTO AO ALCOOLISTA (PAA)}

0 alcoolismo, como uma doença crônica, só foi assim compreendido após vários estudos sobre sua etiologia. Atualmente, está estabelecido seu conceito como doença, observadas as manifestações nos diferentes aspectos da vida do indivíduo, quais sejam, físicas, psíquicas e sociais, representando um grave problema no âmbito da saúde pública. Estima-se que a dependência do álcool atinja de 10\% a 12\% da população mundial ${ }^{1}$ e $11,2 \%$ dos brasileiros que vivem nas
107 maiores cidades do país, segundo levantamento domiciliar sobre o uso de drogas realizado pelo CEBRID².

Dentre as complicações clínicas decorrentes do alcoolismo, têm-se diversas alterações metabólicas e lesões orgânicas. Destacamos aqui as alterações do sistema digestivo (gastrite, esteatose, pancreatite, hepatite alcoólica, cirrose, etc.), cardiovascular (arritmia, hipertensão arterial sistêmica, miocardiopatia alcóolica, etc.), dos sistemas hematopoético, muscular e imunológico, e, por último, a coexistência do alcoolismo e as comorbidades psiquiátricas (depressão, personalidade anti-social, mania, etc.).

Não obstante, são de elevado impacto e custo social os problemas no emprego, com os amigos, legais, policiais, familiares e financeiros, e os casos de violência, previdenciários, entre outros ${ }^{3}$.

De acordo com a Classificação Internacional das Doenças CID-104, 0 alcoolismo situa-se entre os transtornos relacionados às substâncias psicoativas, mais especificamente entre os transtornos mentais e de comportamento decorrentes do uso de álcool, e nas suas respectivas subcategorias, das quais vale destacar a Síndrome de Dependência do Álcool - DAS, que se baseia nos seguintes sinais e sintomas: 1 - empobrecimento do repertório de ingestão, 2 - relevância da bebida, 3 - aumento da tolerância ao álcool, 4 - sintomas repetidos de abstinência, 5 - alívio ou esquiva dos sintomas de abstinência através de mais bebida, 6 - percepção subjetiva da compulsão do beber, 7 reinstalação rápida da tolerância após a quebra da abstinência ${ }^{5}$.

As complicações biopsicossociais, dentre outras, são tomadas como uma das bases para emissão do diagnóstico, podendo ser as mesmas, permanentes ou recuperáveis. Neste sentido, faz-se necessária a elaboração de um modelo de atenção que vise o paciente alcoolista em sua integralidade, já que a intervenção unicamente pautada no plano biológico tem respondido de forma insuficiente às necessidades de saúde destes indivíduos.

Face ao exposto e associado ao desafio de oferecer uma atenção integral ao usuário de álcool, teve início, em 1985, o Programa de Atendimento ao Alcoolista (PAA) do Hospital Universitário Cassiano Antônio Moraes (HUCAM) da Universidade Federal do Espírito Santo (UFES) como estratégia de tratamento interdisciplinar e ambulatorial dirigida a pacientes alcoolistas e seus familiares.

A partir de 1993, esses serviços de referência no estado tornaram-se programas permanentes de extensão, abrindo espaço para novas inserções, fomentando um vasto campo do exercício de ensino, assistência, pesquisa e extensão no cotidiano acadêmico.

A abordagem ao alcoolista é interdisciplinar, buscando a complementariedade nas ações profissionais, tendo em vista 0 problema comum entre as diferentes áreas que assistem ao usuário de álcool e seus familiares. Nesse contexto, a equipe é formada por médico, enfermeiro, assistente social e psicólogo, somando-se ainda a participação de acadêmicos das três primeiras áreas profissionais mencionadas, que cumprem estágio curricular ou atuam como extensionistas.

0 atendimento é realizado em várias etapas, sendo a primeira a Reunião de Sala de Espera, coordenada pelo serviço social, na qual participam todos os pacientes e seus familiares. A duração é de cerca de uma hora, e os pacientes possuem 
liberdade de expressão quanto às suas experiências particulares, sensibilizando os demais participantes quanto à reflexão sobre o alcoolismo-doença e sobre a importância do tratamento, reduzindo a ansiedade. A partir de então, o paciente é atendido pelo Serviço Social de forma individualizada ou junto a familiares para levantamento de sua história de vida, das complicações nas áreas familiares e sociais e confecção da autobiografia.

Segue-se a Consulta Médica, com anamnese, avaliação física, exames laboratoriais de rotina e complementares para alcoolismo. A importância dessa etapa situa-se nas orientações sobre o manuseio da Síndrome de Abstinência (SA) e a intervenção através da internação, quando necessário.

A seguir, é realizada a Consulta de Enfermagem (CE), a qual visa à educação para a saúde do paciente/família com enfoque no alcoolismo-doença. Possui a seguinte estrutura: 1 - Apresentação do PAA (objetivos do programa, atuação da equipe interdisciplinar e papel do enfermeiro), 2 - Avaliação da abstinência (ocorrência de lapsos ou recaídas), 3 - Grupo de ajuda mútua, 4 - SAAD (Short Alcohol Dependence Data, questionário para avaliação da gravidade da Síndrome de Dependência do Álcool), 5 - Teste de Fagerström (questionário para avaliação do grau de dependência à nicotina), 6 Orientações sobre o autocuidado (dar suporte às Necessidades Humanas Básicas afetadas), 7 - Orientações sobre o alcoolismodoença (ampliar a compreensão das causas e conseqüências biopsicossociais), 8 - Medicações (informar sobre os efeitos terapêuticos e colaterais), 9 - Retorno, para continuidade do seguimento e 10 - Encaminhamentos, se necessário, para complementar a atenção integral. São realizadas 06 (seis) CE diárias no PAA-HUCAM-UFES, sendo 03 (três) pacientes de primeira vez e 03 (três) retornos, totalizando 24 atendimentos semanais e 96 atendimentos mensais. No $1^{\circ}$ mês, é realizado o diagnóstico da Síndrome de Dependência do Álcool (SDA); no $2^{\circ}$ mês, são implementadas a manutenção da abstinência alcoólica e a recuperação dos impactos causados pelo álcool (individual, familiar e social), e, a partir do $3^{\circ}$ mês, é estimulada a prevenção da recaída e iniciada sua reinserção social. Os pacientes podem ser atendidos por um ou mais técnicos da equipe e permanecem em seguimento por um período de 12 a 24 meses no Programa, de acordo com a gravidade de cada caso.

Através da metodologia assistencial participativa e conscientizadora adotada no PAA, busca-se a recuperação da saúde física, psíquica e social do indivíduo, investindo na ação educativa, integrando-o como sujeito do processo de tratamento, permitindo a formação de uma relação interpessoal dialógica e horizontal, na qual o usuário seja auxiliado a despertar, a conscientizar-se da sua realidade. É estimulado a reconhecer em si a capacidade de mudança e implementar ações que resultem em melhorias no seu autocuidado e na sua qualidade de vida.

Em síntese, a atenção integral e interdisciplinar proposta pelo PAA permite a prática do ensino, assistência e pesquisa para acadêmicos dos diferentes cursos de graduação na área da saúde - particularmente para a Enfermagem; além disso, proporciona a oportunidade de atuação desses enquanto extensionistas, levando o conhecimento adquirido no contexto universitário de uma forma dinâmica aos usuários e seus familiares. Adquiri-se aí uma vivência além da graduação, que perpassa a grade curricular, acrescentando à formação acadêmica do enfermeiro uma experiência da aplicabilidade prática dos conteúdos teóricos.

\section{NÚCLEO DE ESTUDOS SOBRE O ÁLCOOL E OUTRAS DROGAS (NEAD)}

Os Centros Brasileiros de Referência para tratamento de dependência química, cadastrados na Secretaria Nacional Antidrogas (SENAD), vêm desenvolvendo trabalhos teóricos e práticos relevantes; buscando, desta forma, consolidar os objetivos constantes na Política Nacional Antidrogas da SENAD ${ }^{6}$ e na Política de Atenção Integral aos Usuários de Álcool e outras Drogas ${ }^{7}$ nas áreas temáticas: prevenção, tratamento, recuperação, reinserção social, redução de danos, repressão e estudos-pesquisas-avaliações.

0 Núcleo de Estudos sobre o Álcool e outras Drogas (NEAD) integra os Centros Brasileiros de Referência na área de drogas e foi criado em julho de 1996. Mediante um programa de trabalho interdisciplinar e interinstitucional, promove a produção e divulgação de conhecimentos, a realização de pesquisas, a cooperação técnica e assessoria no campo da dependência química e a colaboração na organização de práticas de saúde que atendam às necessidades da população, respeitando a nova proposta de atenção à saúde da Organização Mundial de Saúde ${ }^{8}$.

No âmbito assistencial, é responsável pela manutenção do Programa de Atendimento ao Alcoolista (PAA), referência estadual no tratamento do alcoolismo ${ }^{9}$, e do Programa de Atendimento ao Tabagista (PAT), implantado a partir de 2006.

Em relação à pesquisa, a equipe técnica tem desenvolvido importantes estudos nas áreas: básica (conhecimentos relativos ao abuso e dependência de drogas e seus efeitos sobre a aprendizagem); clínica-cognitiva (funções cognitivas e disfunções mentais, que estejam ou não sob o tratamento de agentes terapêuticos), clínica (abuso e dependência de drogas e formas de tratamento) e epidemiológica (perfil de uso, levantamento sobre as instituições especializadas em DQ, etc.).

No âmbito da extensão, possui parcerias com organizações governamentais do estado do Espírito Santo, como o Ministério Público do Estado do Espírito Santo (MPES), a Secretaria Estadual de Saúde do Espírito Santo (SESA-ES), a Secretaria Municipal de Saúde de Vitória (SEMUS) e organizações nãogovernamentais, como os grupos de ajuda mútua (AA, ALANON, NA, etc.), para o desenvolvimento de trabalhos conjuntos de capacitação e aperfeiçoamento de recursos humanos, consultoria e assessoria a instituições interessadas na organização de práticas de atenção ao usuário de drogas.

A partir de junho de 2005, o NEAD passou a integrar o Conselho Estadual Antidrogas (COESAD), contribuindo na formulação e implantação da política estadual antidrogas, através da implementação de ações tanto preventivas quanto de tratamento ${ }^{10}$.

0 NEAD é composto por uma equipe interdisciplinar, e suas atividades são desenvolvidas através das coordenadorias de: 
ensino-assistência, pesquisa e extensão. A primeira destina-se a desenvolver ações de atenção a usuários de álcool (PAA) e de tabaco - (PAT, em fase de implantação), possibilitando estágio curricular, extracurricular e monitoria a alunos dos cursos de graduação em Enfermagem, Medicina e Serviço Social, de acordo com os critérios estabelecidos pelos cursos envolvidos. A segunda ocupa-se da realização de investigações científicas básicas, clínicas e epidemiológicas relacionadas ao abuso e dependência de drogas. E a terceira desenvolve consultoria e assessoria a instituições governamentais, ou não, que estejam interessadas na organização de práticas de saúde direcionadas à área de dependência química, bem como promove cursos e eventos científicos para ampliação de conhecimento sobre drogas e a divulgação de resultados de pesquisas e estudos ${ }^{11}$.

Atualmente os seguintes projetos de extensão e pesquisa estão em desenvolvimento pela equipe de Enfermagem:

\section{Sistematização da assistência de enfermagem a pacientes do PAA-HUCAM-UFES}

Visa implantar a consulta de enfermagem, o processo de enfermagem e a visita domiciliar como elementos integradores da atenção a alcoolistas e seus familiares, otimizando a eficiência e eficácia assistencial ${ }^{2}$.

\section{Educação em saúde: um desafio para o cuidado de enfermagem a alcoolistas}

Enfoca a educação em saúde através de ações voltadas para o autocuidado e as necessidades humanas básicas, como papel singular do enfermeiro na atenção a usuários de drogas.

\section{Nas trilhas da produção científica do Núcleo de Estudos sobre o Álcool e outras Drogas}

Visa criar um catálogo com a produção científica do núcleo em 10 anos de experiência;

\section{Atualização das instituições especializadas em dependência química no ES}

Objetiva atualizar o Catálogo das Instituições Especializadas em Dependência Química no ES e auxiliar na consolidação da rede de apoio ao DQ no ES;

\section{Políticas públicas relacionadas às substâncias psicoativas: 0 papel do Estado}

Têm por fim produzir um banco de dados, um catálogo das instituições especializadas em dependência química no ES e analisar a luz das políticas públicas o papel do Estado na área de drogas ${ }^{13}$.

\section{Referências}

1 World Health Organization-WHO. Global status report on alcohol. Geneva; 1999. Disponivel em: http://www.who.int/substance_abuse/pubs_alcohol.htm.

2 Carlini EA, Galduroz JCF, Noto AR, Nappo AS. $1^{\circ}$ Levantamento domiciliar sobre o uso de drogas psicotrópicas no Brasil: estudo envolvendo as 107 maiores cidades do país-2001. São Paulo (SP): Centro Brasileiro de Informações sobre Drogas Psicotrópicas- CEBRID. Departamento de Psicobiologia / Escola Paulista de Medicina; 2002.

3 Ramos SP, Bertolote JM. Alcoolismo hoje. $3^{\mathrm{a}}$ ed. Porto Alegre (RS): Artes Médicas; 1997.
Como vimos, o NEAD tem buscado ampliar sua atuação na sociedade, tanto sob a forma de desenvolvimento de investigações que resultem em evidências científicas sobre a realidade capixaba, quanto inserindo-se no processo de formulação de políticas públicas estaduais.

Em síntese, a complexidade dos problemas que afetam e determinam o estado de saúde da população configura-se num dos desafios fundamentais, especialmente em relação aos determinantes da saúde mental, como a questão das "drogas". Assim, o NEAD tem buscado estabelecer esta ligação entre a universidade-comunidade na consolidação e implementação de propostas preventivas e de intervenção no estado do ES, corroborando o enfrentamento da problemática.

\section{CONSIDERAÇÕES FINAIS}

Devido ao aumento considerável do consumo de drogas nas últimas duas décadas, cada vez mais precoce entre crianças e adolescentes, as substâncias psicoativas tem sido alvo de preocupação da sociedade brasileira, como também os problemas correlacionados, como a criminalidade, os acidentes automobilísticos, os comportamentos anti-sociais e o abandono escolar, entre outros. Além disso, a falta de políticas públicas em longo prazo para atender integralmente os usuários de drogas tem resultado numa crescente demanda por serviços de tratamento.

A comunidade científica tem buscado participar do enfrentamento da problemática das drogas, desenvolvendo pesquisas que ampliem a compreensão e as evidências científicas sobre a temática. Nesse sentido, o NEAD e o PAA voltam seus esforços para otimizarem suas ações de ensinoassintência-pesquisa-extensão, reafirmando sua importância na sociedade capixaba, não só como integrante da rede de apoio ao dependente químico no Estado, como também pela sua produção de conhecimento na área de drogas. Todavia, é necessária uma maior atuação na formação de recursos humanos e ampliação dos trabalhos técnicos que possibilitem a criação de novos programas e/ou serviços que atendam as demandas do dependente químico no Estado.

Concluindo, é importante ressaltar a magnitude dos trabalhos desenvolvidos na UFES, tanto na qualidade de programas permanentes de extensão universitária como de projetos político-pedagógicos, fazendo deles um elo fundamental entre a universidade e a comunidade.

40rganização Mundial da Saúde-OMS. Classificação de transtornos mentais e de comportamento-CID 10: descrições clínicas e diretrizes diagnósticas. Tradução de Dorgival Caetano. Porto Alegre (RS): Artes Médicas; 1993. 5 Edwards G. 0 tratamento do alcoolismo. Porto Alegre (RS): Artmed; 1999.

6 Ministério da Justiça (BR). Política Nacional Antidrogas. Brasília (DF); 2001.

7 Ministério da Saúde (BR). A política do Ministério da Saúde para atenção integral a usuários de álcool e outras drogas. 1ª ed. Brasília (DF);2003. 
8 Organização Mundial da Saúde-OMS. Relatório sobre a saúde no mundo 2001 - saúde mental: nova concepção, nova esperança. Genebra; 2001. 9 Macieira MS, Gomes MPZ, Garcia MLT. Programa de Atendimento ao Alcoolista do Hospital Universitário Cassiano Antônio Moraes da Universidade Federal do Espírito Santo -PAA-HUCAM-UFES. J Bras Psiquiatr 1993; 42 (2): 97-109.

10 Vitória (ES). Secretaria Estadual de Justiça. Fórum Estadual Antidrogas. $2^{\circ}$ ed.Vitória (ES): SEJUS; 2001.
11 Macieira MS, Gomes MPZ, Garcia MLT, Palacios EMN. Núcleo de estudos sobre 0 alcool e outras drogas. Rev HUCAM 2002; 11: 11-15.

12 Barros JFV, Camata MW, Santos ES, Macieira MS. Metodologia da assistência de enfermagem desenvolvida com pacientes alcoolistas do programa de atendimento ao alcoolista. J Bras Psiquiatr 2000; 49 (07): 247-54.

13 Siqueira MM. Políticas públicas relacionadas às substâncias psicoativas: o papel do estado. Vitória (ES): UFES-UNIFESP; 2004. 